\title{
Treatment with Anti-HMGB1 Monoclonal Antibody Does Not Affect Lupus Nephritis in MRL/Ipr Mice
}

\author{
Fleur Schaper, ${ }^{1}$ Mirjan M van Timmeren, ${ }^{2}$ Arjen Petersen, ${ }^{2}$ Gerda Horst, ${ }^{1}$ Marc Bijl, ${ }^{3}$ Pieter C Limburg, ${ }^{4}$ \\ Johanna Westra, ${ }^{1}$ and Peter Heeringa ${ }^{2}$
}

${ }^{1}$ Department of Rheumatology and Clinical Immunology, University Medical Center Groningen, University of Groningen, Groningen, the Netherlands; ${ }^{2}$ Department of Pathology and Medical Biology, University Medical Center Groningen, University of Groningen, Groningen, the Netherlands; ${ }^{3}$ Department of Internal Medicine and Rheumatology, Martini Hospital, Groningen, the Netherlands; and ${ }^{4}$ Department of Laboratory Medicine, University Medical Center Groningen, University of Groningen, Groningen, the Netherlands

\begin{abstract}
High mobility group box 1 (HMGB1) is a nuclear DNA binding protein that acts as an alarmin when secreted. HMGB1 is increased in systemic lupus erythematosus and might represent a potential therapeutic target. We investigated whether treatment with an anti-HMGBI antibody affects the development of lupus nephritis in MRL/Ipr mice. Seven-week-old MRL/lpr mice were injected intraperitoneally twice weekly for 10 wks with $50 \mu \mathrm{g}$ monoclonal anti-HMGB1 (2G7, mouse lgG2b) ( $\mathrm{n}=12$ ) or control antibody $(n=11)$. Control MRL/MPJ mice $(n=10)$ were left untreated. Every 2 wks, blood was drawn and urine was collected at wk 7, 11 and 17. Mice were sacrificed at 17 wks for complete disease evaluation. Plasma HMGB1 and anti-HMGB1 levels were increased in MRL/Ipr mice compared with control MRL/MPJ mice. There were no differences in allbuminuria, urine HMGB1 and plasma levels of complement C3, anti-dsDNA and proinflammatory cytokines between untreated and treated mice at any time point. Lupus nephritis of mice treated with anti-HMGBI monoclonal antibody (mAb) was classified as class III $(n=3)$ and class IV $(n=9)$, while mice treated with control mAb were classified as class II $(n=4)$, class III $(n=2)$ and class IV $(n=5)$. IgG and C3 deposits in kidneys were similar in mice treated with anti-HMGB1 mAb or control mAb. In conclusion, treatment with monoclonal anti-HMGB-1 antibody 2G7 does not affect development of lupus nephritis, disease progression or proinflammatory cytokine levels in MRL/Ipr mice. This result indicates that blocking of HMGB1 by this neutralizing antibody does not affect lupus nephritis in MRL/Ipr mice.
\end{abstract}

Online address: http://www.molmed.org

doi: $10.2119 /$ molmed.2015.00176

\section{INTRODUCTION}

Systemic lupus erythematosus (SLE) is a systemic autoimmune disease characterized by presence of autoantibodies against nuclear components, leading to circulating immune complexes that are deposited in organs, causing tissue damage. SLE can affect multiple organs, including skin, joints and kidneys. Glomerulonephritis leading to persistent proteinuria and chronic renal failure is one of the most severe complications and is associated with significant mortality (1).

High mobility group box 1 (HMGB1) is a nonhistone nuclear protein that has a dual function. Inside the cell, it is ubiquitously expressed in the nucleus where it binds to DNA. HMGB1 can bend promoter regions and interacts directly with nucleosomes, thereby altering the

Address correspondence to Johanna Westra, Department of Rheumatology and Clinical Immunology, AA21, University Medical Center Groningen, Hanzeplein 1, 9713 GZ, Groningen, the Netherlands. Phone: +31-50-36 1-2945; Fax: +31-50-361-9308; E-mail: johanna.westra@umcg.nl.

Submitted July 29, 2015; Accepted for publication January 12, 2016; Published Online (www.molmed.org) January 13, 2016.

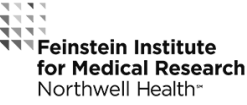
Feinstein Institute
for Medical Research Northwell Health 
disease activity (10-14). It has been hypothesized that impaired clearance and accumulation of apoptotic debris in SLE might lead to increased HMGB1 levels locally and systemically. Furthermore, higher levels of HMGB1 were observed in serum from patients with renal involvement compared with patients without renal involvement $(10,13)$. Kidney biopsies from patients with lupus nephritis showed strong expression of HMGB1 at cytoplasmic and extracellular sites, suggesting active release of HMGB1 (11). Collectively, these studies suggest that HMGB1 is an important factor in the pathogenesis of SLE and that it represents a potential therapeutic target. The MRL/MpOlaHsd-Tnfrsf6 ${ }^{\text {lpr }}$ (MRL/lpr) mouse is a frequently used mouse strain that develops a severe spontaneous autoimmune disease similar to SLE (15). Mice homozygous for the lymphoproliferation spontaneous mutation (Fas ${ }^{\mathrm{lpr}}$ ) show loss of Fas function leading to a defect in Fas-mediated apoptosis. On the MRL genetic background, this leads to extensive lymphoproliferation and generation of autoreactive T cells. The ensuing disease is characterized by lymphadenopathy, autoantibody production, complement activation and immune complex glomerulonephritis that usually manifests between 14 and 18 wks of age (16-18).

We hypothesized that HMGB1 represents a potential therapeutic target in SLE and that inhibition of HMGB1 will attenuate renal involvement in SLE. In this study, we therefore investigated whether treatment with a neutralizing monoclonal anti-HMGB1 antibody could beneficially affect the development of lupus nephritis in MRL/lpr mice.

\section{MATERIALS AND METHODS}

\section{Mice}

Five-week-old female MRL/lpr mice were obtained from Harlan, and 5-wk-old female MRL/MPJ were obtained from The Jackson Laboratory. Mice were grouphoused in a temperature and humiditycontrolled environment with a 12-h light-dark cycle. Animals were allowed ad libitum access to drinking water and standard chow. Animal experiments were approved by the local animal care and experimentation committee (DEC 6464A).

\section{Neutralizing Anti-HMGB 1 Antibody}

Monoclonal anti-HMGB1 antibody (clone 2G7, IgG2b, a gift from Kevin Tracey at the Feinstein Institute for Medical Research) was used for treatment in MRL/lpr mice. This antibody has been extensively characterized previously with respect to its HMGB1 neutralizing activity in in vitro and in vivo studies. The neutralizing activity of anti-HMGB1 monoclonal antibody (mAb) has been tested in cell culture assays, with both human and mouse macrophages, and in animal models of HMGB1-mediated damage, such as sepsis (8,19-24). Moreover, anti-HMGB1 $\mathrm{mAb} 2 \mathrm{G} 7$ has been shown to neutralize the cytokine isoform of HMGB1 (19).

\section{Treatment of the Mice with Anti-HMGB 1 Antibody}

Ten MRL/lpr mice were followed from wk 7 until wk 17 without any intervention. Two groups of $12 \mathrm{MRL} / \mathrm{lpr}$ mice were injected intraperitoneally twice weekly, for 10 wks, with $50 \mu \mathrm{g} /$ mouse monoclonal anti-HMGB1 antibody (clone 2G7, IgG2b) or control IgG2b antibody (BioXCell). A control group of MRL/MPJ mice $(n=10)$ was left untreated. Before start of treatment, at wk 7, blood and urine was collected. Then, every 2 wks, plasma (ethylenediaminetetraacetic acid) was collected from the saphenous vein. Urine was collected during $18 \mathrm{~h}$ at 7, 11 and $17 \mathrm{wks}$. At death, spleens and kidneys were harvested. One half of each kidney was fixed in formalin and embedded in paraffin; the remaining half was snap-frozen in liquid $\mathrm{N}_{2}$ and stored at $-80^{\circ} \mathrm{C}$.

\section{Laboratory Measurements}

Automated total and differential white blood cell (WBC) counts were determined in whole blood obtained at death using the Sysmex XT-1800iV (Sysmex Nederland BV). Plasma levels of complement C3 (GenWay Biotech) and anti-dsDNA IgG (Alpha Diagnostic International) were measured by enzyme-linked immunosorbent assay (ELISA), according to the manufacturers' instructions. Serum blood urea nitrogen (BUN) levels were determined by the Quantichrom DIUR 500 kit (BioAssay Systems). Albuminuria was determined by ELISA (Bethyl Laboratories) according to the manufacturer's instructions. Plasma levels of the cytokines interleukin (IL)-4, IL-6, IL-17A, interferon (IFN)- $\alpha$ and tumor necrosis factor (TNF)- $\alpha$ were measured with a Multiplex panel (ProcartaPlex Mouse Simplex, Affymetrix eBioscience) according to the manufacturer's instructions. Samples were measured by using the Luminex 100 System, and data were analyzed with StarStation software, version 2.3 (Applied Cytometry). Plasma levels of anti-HMGB1 were measured by an in-house developed ELISA. In short, Costar polystyrene plates were coated overnight with $1 \mu \mathrm{g} / \mathrm{mL}$ recombinant HMGB1 (Sigma). Mouse plasma samples were added in duplicate at 500-fold or 7,500-fold dilutions (for anti-HMGB1-treated mice). Detection of antibodies was done with rabbit anti-mouse immunoglobulin G-horseradish peroxidase (IgG-HRP) (Dako). Levels of anti-HMGB1 were calculated against a standard curve of a known concentration of monoclonal anti-HMGB1 antibody (2G7, mouse IgG2b).

Plasma and urine levels of HMGB1 were measured by Western blotting as described previously (10), by using polyclonal anti-HMGB1-biotin (Thermo Fisher Scientific) for detection.

\section{Gene Expression Analysis by Quantitative Reverse Transcriptase-Polymerase Chain Reaction}

Total RNA was extracted from 20 10- $\mu$ m-thick kidney cryosections by using the RNeasy Mini Plus Kit (Qiagen) according to the manufacturer's instructions. RNA yield, cDNA synthesis and quantitative polymerase chain reaction amplifications were performed as described previously (25). 
The following Assay-On-Demand primers were used: glyceraldehyde 3-phosphatedehydrogenase (GAPDH); monocyte chemoattractant protein-1 (MCP-1), kidney injury molecule-1 (KIM-1), neutrophil gelatinase-associated lipocalin (NGAL), IL-6 and TNF- $\alpha$. The amount of target was normalized to an endogenous reference (GAPDH) and expressed as relative expression $\left(2^{-\Delta \mathrm{CT}}\right)$.

\section{Renal Histopathology}

The 2- $\mu \mathrm{m}$ paraffin kidney sections were routinely stained with hematoxylin and eosin (H\&E) and periodic acid-Schiff (PAS). The slides were evaluated in a blinded manner by an expert renal pathologist, and kidney pathology was evaluated using the lupus nephritis classification system, as described by Weening et al. (26). HMGB1 staining was performed as described previously (11).

Complement C3 and IgG staining was performed on $5-\mu$ m frozen kidney sections with $1 \mu \mathrm{g} / \mathrm{mL}$ rabbit anti-C3 antibody (Thermo Fisher Scientific) followed by goat-anti-rabbit IgG-HRP. For IgG, rabbit anti-mouse IgG-HRP was used. Peroxidase activity was detected with 3,3'-diaminobenzidine, and sections were counterstained with Mayer hematoxylin. All sections were scored digitally after examination by using a Nanozoomer Digital Pathology Scanner (NDP Scan U10074-01, Hamamatsu Photonics) and quantified [(number of positive cells $\times 0.5$ ) + number of strong positive cells/total cells] with software of Aperio ImageScope (V11.2.0.780 Aperio Technologies).

\section{Statistics}

Data were analyzed by using SPSS (version 22, IBM). Data are expressed as median and range and were analyzed by using Mann-Whitney $U$ test or Kruskal-Wallis test. For noncontinuous variables, a Fisher exact test was used. Generalized estimating equations were used to analyze changes over time within mice, and the exchangeable matrix correlation structure was used. When residuals were not normally distributed, parameters were transformed (square root or logarithmic) before being entered in the equation. $p$ values $<0.05$ were considered statistically significant.

All supplementary materials are available online at www.molmed.org.

\section{RESULTS}

\section{Plasma HMGB 1 and Anti-HMGB 1 Levels Increase over Time in Lupus-Prone MRL/Ipr Mice}

We first analyzed plasma levels of HMGB1 by Western blotting in a separate cohort of $10 \mathrm{MRL} / \mathrm{lpr}$ mice from 6 wks until 17 wks of age. As shown in Figure 1, plasma HMGB1 (Figure 1A) and anti-HMGB1 (Figure 1B) were clearly detectable in 6-week-old mice. Moreover, plasma HMGB1 levels increased with age and disease progression. These results indicate that MRL/lpr mice have increasing plasma levels of HMGB1 over time and therefore represent an appropriate model to investigate whether intervention with an anti-HMGB1 antibody has beneficial effects on the development of lupus nephritis.

\section{HMGB 1 mAb Treatment Does Not Affect Plasma Parameters of Disease Activity}

Plasma levels of anti-dsDNA and complement C3 were analyzed at four time points in both MRL/lpr groups and MRL/MPJ mice (Figures 2A, B) by generalized estimating equations (GEE) analysis. At all time points, starting at wk 7, MRL/lpr mice had significantly higher levels of anti-dsDNA than MRL/MPJ mice $(p<0.001$, Figure 2A). In MRL/lpr mice levels of anti-dsDNA increased over time and were highest at wk 17, whereas in MRL/MPJ mice the levels of anti-dsDNA increased until wk 13 and remained at a similar level at wk 17. Levels of anti-dsDNA were similar in MRL/lpr mice treated with anti-HMGB1 mAb or control $\mathrm{mAb}(p=0.09$, Figure 2B). In MRL/lpr as well as MRL/MPJ mice, complement C3 levels decreased over time $(p<0.001)$ and were lowest at wk 17. Overall, complement C3 was significantly decreased in MRL/lpr compared with MRL/MPJ $(p<0.01)$, but was not different between mice treated with anti-HMGB1 $\mathrm{mAb}$ and control $\mathrm{mAb}$ ( $p=0.55$, Figure 2B).

Plasma levels of HMGB1 were analyzed at six time points (Figure 2C). GEE analysis demonstrated that HMGB1 significantly increased over time in all three groups $(p<0.001)$. As expected, at all time points, MRL/lpr mice had higher levels of HMGB1 than MRL/MPJ mice $(p<0.05)$; however, no significant differences were observed between MRL/lpr mice treated with anti-HMGB1 $\mathrm{mAb}$ or control mAb $(p=0.32)$.

Plasma levels of anti-HMGB1 were measured at six time points by ELISA (Figure 2D). Anti-HMGB1 significantly increased over time in MRL/lpr mice $(p<0.001)$, but not in MRL/MPJ mice. At wk 7, before start of treatment in MRL/lpr mice, plasma levels of anti-HMGB1 were similar in all MRL/lpr and MRL/MPJ mice. At all of the time points, anti-HMGB1 levels were substantially higher in MRL/lpr mice treated with anti-HMGB1 $\mathrm{mAb}$ than with control mAb $(p<0.001$, Figure 2D). In MRL/MPJ mice, levels of anti-HMGB1 did not change over time, whereas in MRL/lpr mice treated with control mAb, anti-HMGB1 levels gradually increased until wk 15, after which they stabilized.

Plasma levels of several proinflammatory cytokines were analyzed with a multiplex assay, as shown in Figure 3. At wk 7, the levels of IFN- $\alpha$, IL-17A and IL-6 were similar in MRL/lpr and MRL/MPJ mice, whereas TNF- $\alpha$ was higher in MRL/lpr mice $(p<0.001)$. IL-6 levels (Figure 3A) increased over time in MRL/MPJ as well as in MRL/lpr mice; however, no differences were observed between MRL/lpr mice treated with anti-HMGB1 mAb or control mAb. IL-17A levels (Figure 3B) decreased over time in MRL/MPJ as well as MRL/lpr mice; however, no differences were observed between treated groups. In MRL/lpr mice, 
A

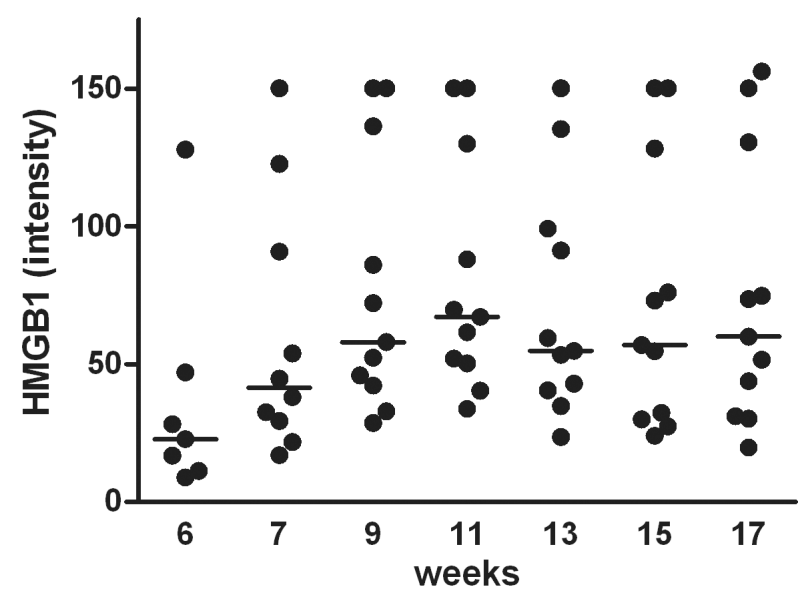

B

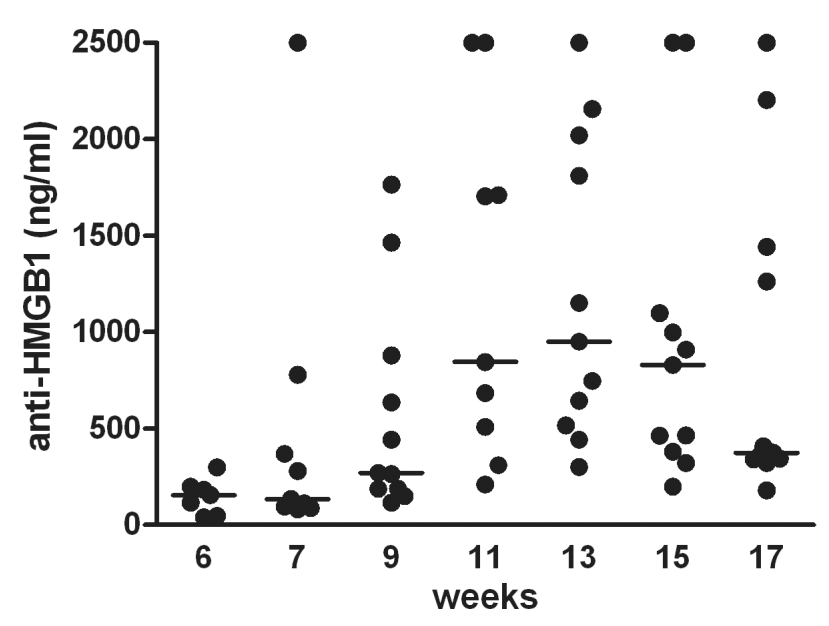

Figure 1. Plasma HMGB1 and anti-HMGB1 antibody levels increase with age in MRL/lpr mice. (A) Plasma HMGB1 levels were measured by Western blot at wks 6, 7, 9, 11, 13, 15 and 17 in MRL/lpr mice $(n=11)$. HMGB1 levels are presented as values of fluorescence intensity and were normalized against a standard of Jurkat cell lysate. (B) Plasma anti-HMGB levels $(\mathrm{ng} / \mathrm{mL})$ were measured by ELISA at wks $6,7,9,11,13,15$ and 17 in MRL/Ipr mice $(n=11)$. Anti-HMGB 1 levels are presented as values of fluorescence intensity and were normalized against a standard of monoclonal anti-HMGB1 antibody.

but not in MRL/MPJ mice, IFN- $\alpha$ (Figure 3C) and TNF- $\alpha$ (Figure 3D) levels increased significantly $(p<0.01)$ over time; however, no differences were observed between MRL/lpr mice treated with anti-HMGB1 mAb or mice treated with control mAb. To summarize, although MRL/lpr mice treated with anti-HMGB-1 mAb displayed massively increased circulating anti-HMGB-1 antibody levels, no effect was observed on plasma levels of HMGB1, anti-dsDNA, complement C3 and proinflammatory cytokines.

\section{HMGB 1 mAb Treatment Does Not Affect Body and Spleen Weight and Distribution of WBCs}

To investigate whether treatment with anti-HMGB1 had an effect on body weight, mice were weighed every week. As shown in Supplementary Figure S1A, all three groups of mice had increasing body weight from wk 7 until death at wk 17, and no differences between the groups were observed. As expected, average spleen weight at death was increased in MRL/lpr mice compared with MRL/ MPJ mice $(p<0.0001)$; however, average spleen weight was similar between MRL/lpr mice treated with anti-HMGB1 $\mathrm{mAb}$ or control mAb (Supplementary Figure S1B). Total WBC count and differential WBC counts were also similar between anti-HMGB1 and control mAbtreated MRL/lpr mice (Supplementary Table S1). These results demonstrate that treatment with monoclonal anti-HMGB1 antibody does not influence body and spleen weight or WBC composition.

\section{HMGB 1 mAb Treatment Does Not Affect Kidney Pathology and Proteinuria}

Kidney pathology was evaluated by using the lupus nephritis classification system, as described by Weening et al. (26). MRL/MPJ mice, as expected, did not show any lupus nephritis, whereas both groups of MRL/lpr did (Figure 4A). Lupus nephritis of MRL/lpr mice treated with anti-HMGB1 mAb was classified as class III $(n=3)$ and class IV $(n=9)$, whereas mice treated with control $\mathrm{mAb}$ were classified as class II $(n=4)$, class III $(n=2)$ and class IV $(n=5)$. In all mice, mostly proliferative lesions were found, and all displayed mononuclear cell infiltration. In both groups, two mice showed vasculitis. In addition, chronicity was low, and two mice in both groups showed sclerosing lupus nephritis. None of the mice developed tubulointerstitial disease, or glomerular and/or fibrous crescents.

Renal deposits of IgG and complement C3 were determined by immunohistochemistry (Figures 4A, B). As expected, extensive glomerular IgG and C3 deposits were detected in MRL/lpr mice when compared with the control MRL/MPJ mice. However, the extent of glomerular IgG and C3 deposits was similar in MRL/lpr mice treated with anti-HMGB1 mAb 
A

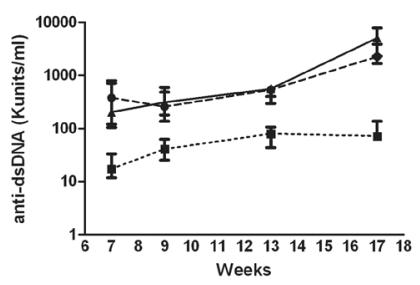

C

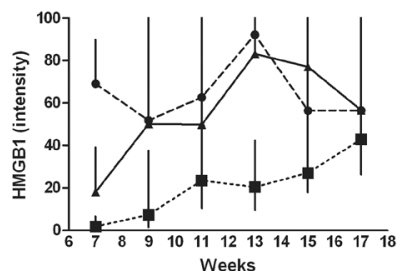

B

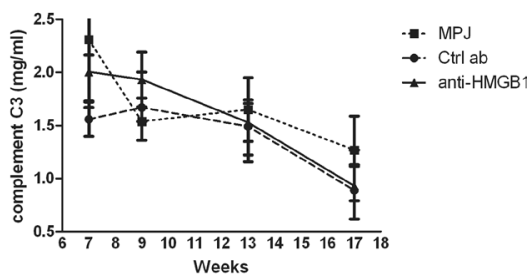

D

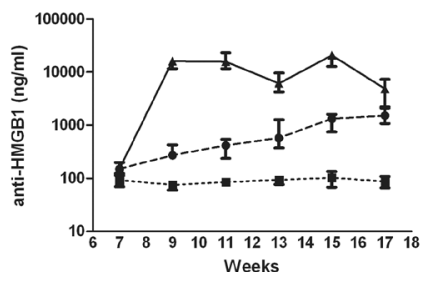

Figure 2. Anti-HMGBI mAb treatment of MRL/lpr mice does not affect plasma levels of HMGB1, anti-dsDNA antibodies and complement C3, while increasing HMGB1 antibody levels. (A) Plasma anti-dsDNA (units $/ \mathrm{mL}$ ) was measured by ELISA in MRL/MPJ $(n=10)$ and MRL/Ipr ( $\mathrm{n}=10-12)$ mice at wks 7, 9, 13 and 17. (B) Plasma complement C3 (mg/mL) was measured by ELISA in MRL/MPJ $(n=10)$ and MRL/Ipr $(n=10-12)$ mice at wk $7,9,13$ and 17. (C) Plasma HMGB1 was measured by Western blotting in MRL/MPJ $(n=10)$ and MRL/Ipr $(n=10-12)$ mice at wks $7,9,11,13,15$ and 17. HMGB1 levels are presented as values of fluorescence intensity and were normalized against a standard of Jurkat cell lysate. (D) Plasma anti-HMGB1 ( $\mathrm{ng} / \mathrm{mL}$ ) was measured by ELISA in MRL/MPJ $(\mathrm{n}=10)$ and MRL/Ipr $(n=10-12)$ mice at wks 7,9, 11, 13, 15 and 17. Anti-HMGB1 levels are presented as values of fluorescence intensity and were normalized against a standard of monoclonal anti-HMGBI antibody. Depicted are the median values with range at each time point.

or control mAb. As expected, HMGB1 staining showed nuclear staining in kidneys from MRL/MPJ mice (Supplementary Figure S2). In kidneys from MRL/lpr mice, extracellular HMGB1 was observed. Moreover, there were also HMGB1-negative nuclei (blue staining) present, which suggests active release of HMGB1 from these cells, but there was no difference between mice treated with anti-HMGB1 $\mathrm{mAb}$ or control mAb.

Renal function was determined by measuring BUN levels at wks 7, 11 and 17 (Figure 5A). At wk 7, BUN levels were similar in MRL/lpr and MRL/MPJ mice, while at wks 11 and 17, BUN levels were increased in both groups (MRL/lpr mice compared with MRL/MPJ mice) $(p<0.01)$. There were no differences in BUN levels between mice treated with anti-HMGB1 mAb or control mAb.
Renal injury was also evaluated by 18 -h albumin excretion into the urine (Figure 5B). At wks 7 and 11, urinary albumin excretion was similar in MRL/lpr and MRL/MPJ mice. At wk 17, urinary albumin excretion was highly increased in the MRL/lpr mice but not in MRL/MPJ mice $(p<0.0001)$. However, albuminuria was similar in MRL/lpr mice treated with anti-HMGB1 mAb or control mAb. Urine HMGB1 levels were analyzed by Western blotting (Figure 5C). At wks 7 and 11, urine HMGB1 was undetectable in all mice. At wk 17, urine HMGB1 levels were increased in MRL/lpr mice; however, no difference between treated groups was seen. To summarize, MRL/lpr mice displayed renal injury characteristic of lupus nephritis, which was unaffected by treatment with anti-HMGB1 mAb.

\section{HMGB 1 mAb Treatment Does Not Affect Proinflammatory Cytokine and Injury-Related Gene Expression in the Kidney}

To further investigate renal inflammation, mRNA levels of the proinflammatory cytokines IL- 6 , TNF- $\alpha$ and MCP-1 and of renal injury-related biomarkers NGAL and KIM-1 were assessed in kidneys (Figure 6). As expected, levels of IL- 6 , TNF- $\alpha$, NGAL and MCP-1 were higher in MRL/lpr mice compared with MRL/MPJ mice $(p<0.05)$. However, KIM-1 levels (Figure 6E) were comparable between MRL/MPJ and MRL/lpr mice, confirming renal histology in which no tubulointerstitial disease was observed. No differences were found in expression levels of all investigated genes between MRL/lpr mice treated with anti-HMGB1 mAb or control mAb (Figure 6), confirming the results of renal histology that treatment with anti-HMGB1 mAb does not influence kidney damage.

\section{DISCUSSION}

Lupus nephritis is one of the most severe complications of SLE and is associated with significant mortality. In SLE, HMGB1 serum levels are associated with disease activity and renal involvement; therefore, it was proposed that HMGB1 might represent a potential therapeutic target in SLE $(11,12,27)$. By using the lupus-prone MRL/lpr mouse model, we show that administration of neutralizing monoclonal anti-HMGB1 antibodies does not affect development of lupus nephritis, disease progression or proinflammatory cytokine expression.

In accordance with observations in patients with SLE (10-14), plasma HMGB1 levels were increased in lupus-prone MRL/lpr mice compared with the control MRL/MPJ mice that do not develop disease. Moreover, HMGB1 levels increased with disease progression, which again is in agreement with observations in SLE patients where serum HMGB1 levels correlate with the SLE disease activity index $(10,14)$. Upon development of lupus nephritis 
A

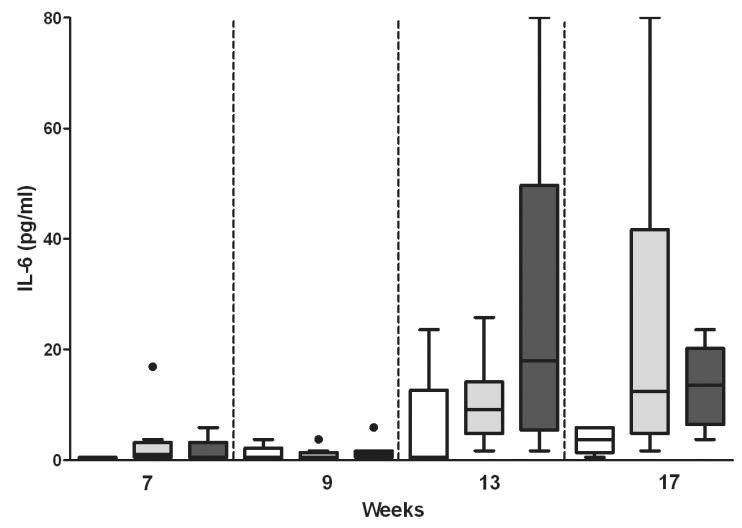

C

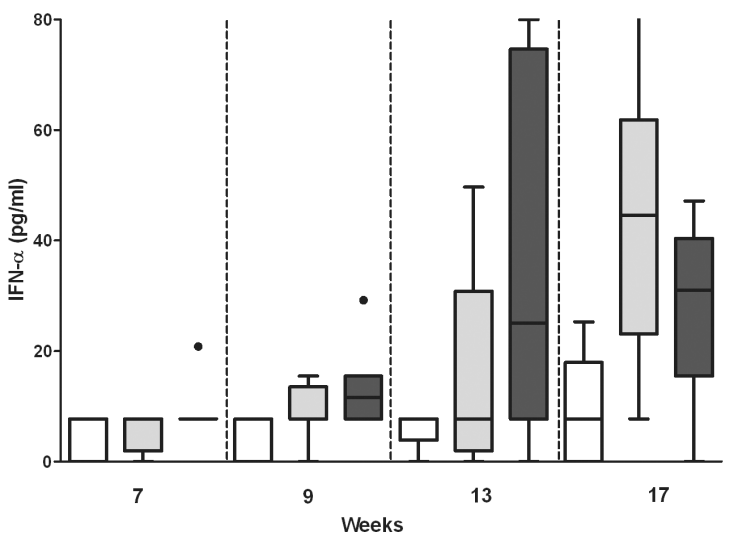

B

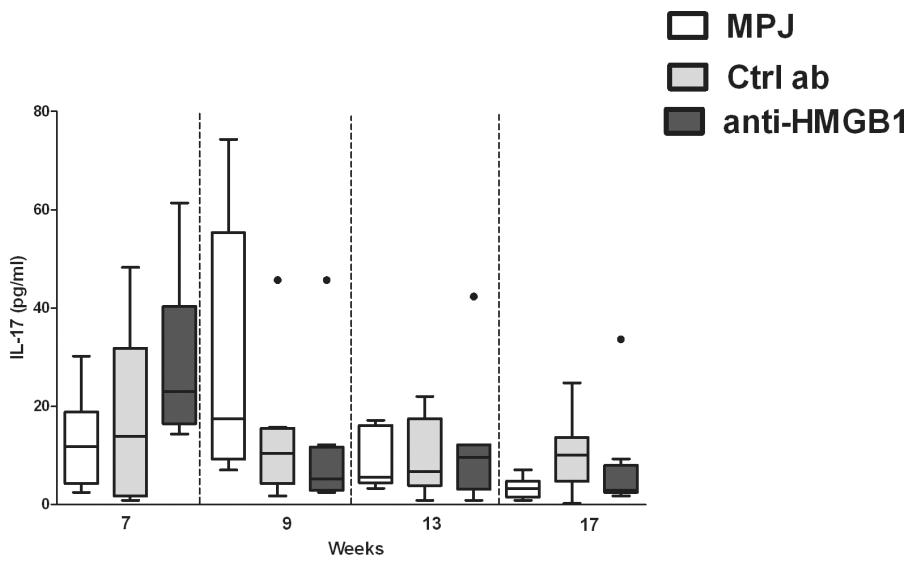

D

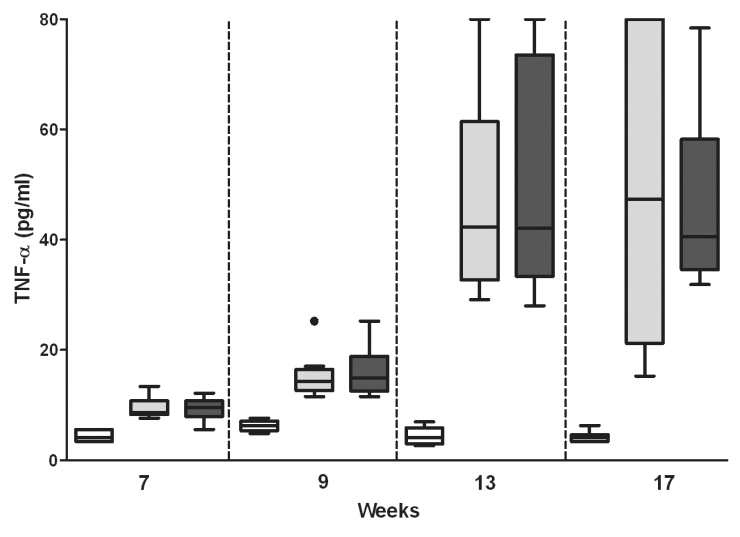

Figure 3. Anti-HMGB1 mAb treatment of MRL/lpr mice does not affect plasma levels of proinflammatory cytokines. Plasma levels of the cytokines IL-6 (A), IL-17A (B), IFN- $\alpha$ (C) and TNF- $\alpha$ (D) were measured by multiplex in MRL/MPJ and MRL/Ipr mice at wks 7, 9, 13 and 17. Box and whiskers plot, median and interquartile range are shown for $n=6-8$ mice per group. Separate dots indicate outliers.

at wk 17, HMGB1 was also detectable in urine, as was also shown in patients (11). In line with renal HMGB1 immunohistochemistry in patients, we observed HMGB1 expression at extracellular sites in kidneys from MRL/lpr mice, which suggests active release of HMGB1. Collectively, these data indicate that HMGB1 release occurs in the MRL/lpr mouse model and that, also with respect to HMGB1, this mouse model reflects human disease.

However, as we describe here, intraperitoneal administration of the monoclonal anti-HMGB1 antibody 2G7 to lupus-prone MRL/lpr mice (50 $\mu \mathrm{g} /$ mouse twice weekly from wk 7 until wk 17) did not ameliorate development of lupus nephritis. Previously, the monoclonal anti-HMGB1 antibody
2G7 has been successfully used in various models of inflammation in which a proinflammatory effect of HMGB1 was suspected. For example, intraperitoneal administration of $\mathrm{mAb} 2 \mathrm{G} 7$ in a mouse model of rheumatoid arthritis ameliorated development of arthritis. In this specific study, either $100 \mu \mathrm{g} /$ mouse was injected every second day for $5 \mathrm{wks}$ or $70 \mu \mathrm{g} /$ mouse daily for $1 \mathrm{wk}(7,28)$. Moreover, intraperitoneal administration of a single dose of mAb $2 \mathrm{G} 7(10 \mu \mathrm{g} /$ mouse $)$ $24 \mathrm{~h}$ after cecal ligation and puncture significantly improved survival in this sepsis model (29). In addition, mAb 2G7 has been shown to prevent TLR4-mediated early graft failure after pancreatic islet graft transplantation; however, in this specific study, the antibody (50 $\mu \mathrm{g} /$ mouse) was injected into the portal vein of the liver together with the transplanted islets (28). Collectively, these studies demonstrate that the anti-HMGB1 mAb 2G7, in comparable dosages and using similar administration routes, confers protective effects in various inflammatory disease models.

In addition, a number of studies, albeit using different anti-HMGB1 antibodies that also recognize a different epitope, have shown beneficial effects of treatment with anti-HMGB1 antibodies in mouse models of sepsis and renal ischemia/ reperfusion $(8,30)$. In these models, it was demonstrated that HMGB1 is an early mediator of damage and that neutralization of HMGB1 inhibits downstream effects, such as release of proinflammatory cytokines. However, these models represent reversible acute disease models, 
A

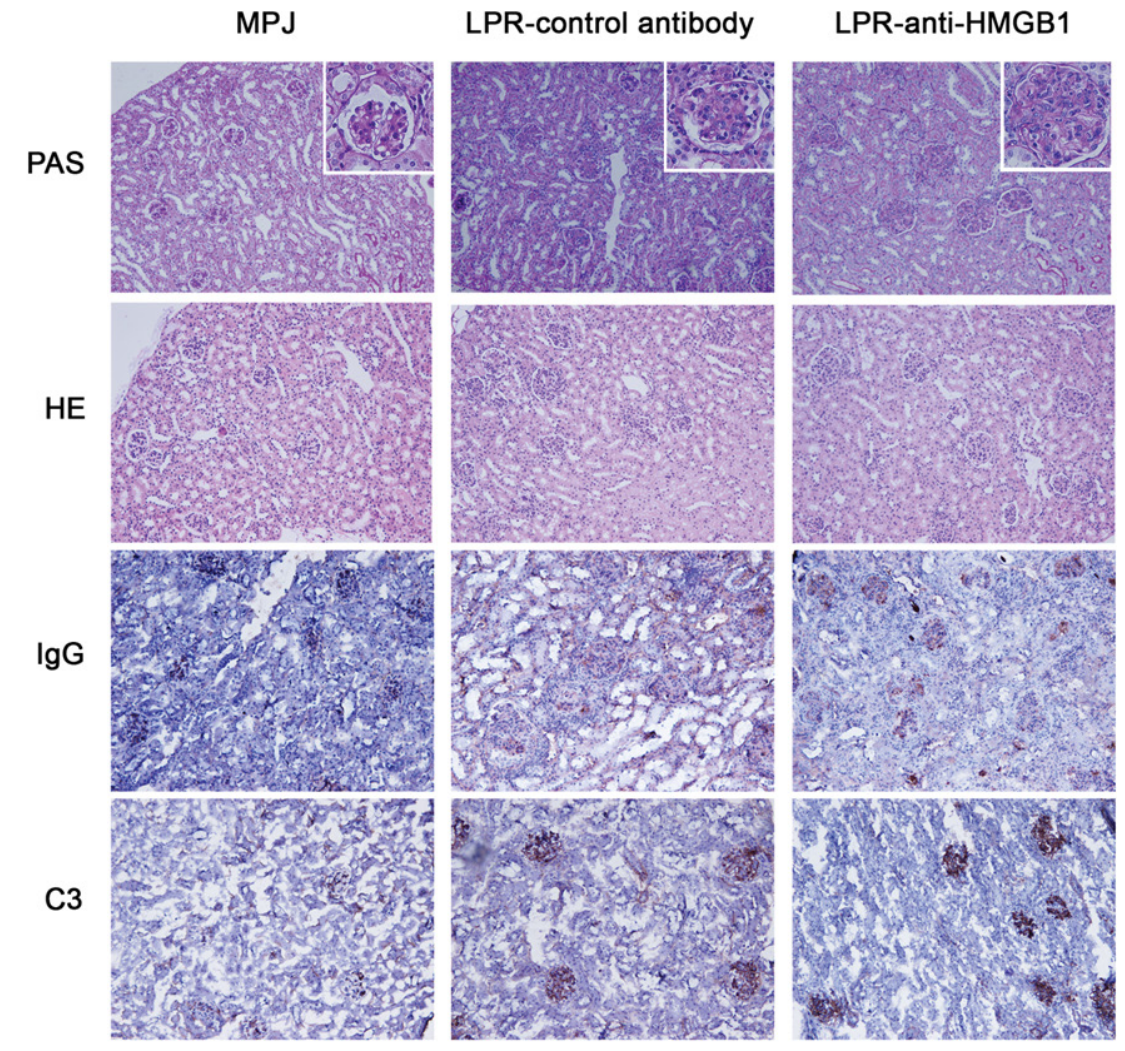

B

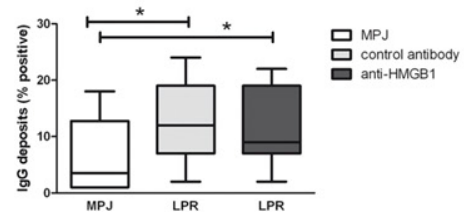

of arthritis $(31,32)$ and sepsis $(8)$, administration of Box A exerted beneficial effects on disease progression. It should be noted, however, that RAGE is only one of the receptors for HMGB1, and hence it is likely that not all effects of HMGB1 will be inhibited when using Box A.

In addition, Li et al. recently reported that blocking serum HMGB1 with glycyrrhizin, a traditional herbal medicine that blocks HMGB1, alleviated lupus nephritis induced in mice by activated lymphocyte-derived DNA (ALD-DNA) (33). In this model, lupus nephritis is induced by active immunization of mice with DNA from lymphocytes that have undergone activation-induced cell death (ALD-DNA) in complete Freund's adjuvant. After induction, serum HMGB1 levels gradually increased. Glycyrrhizin treatment attenuated HMGB1 as well as circulating proinflammatory cytokine levels and alleviated renal pathology. However, for glycyrrhizin, a range of biological effects were reported, including antiinflammatory, antioxidant, free radical-scavenging, antiulcer, antihepatotoxic, antimicrobial, cytoprotective and cytotoxic activities $(34,35)$. Therefore, it is unclear whether glycyrrhizin exerts its beneficial effects solely via blocking of HMGB1 in this ALD-DNA-induced lupus mouse model.

The results presented here appear

Figure 4. Anti-HMGBI mAb treatment of MRL//pr mice does not affect renal pathology and renal immune complex deposition. (A) Representative pictures of PAS, H\&E, C3 and IgG staining in kidney sections of 17-wk-old MRL/MPJ and MRL/Ipr mice (10x). Inserts show a glomerulus in detail (40x). (B) Quantitative analysis of $\mathrm{C} 3$ and $\lg G$ deposition in kidney sections of 17-wk-old MRL/MPJ and MRL/Ipr mice. Box and whiskers plot, median and interquartile range are shown for $n=8-11$ mice per group. A Kruskal-Wallis test was used to test for overall differences between the three groups. To investigate which group was different, further testing was performed to compare groups separately using a Mann-Whitney U test. * $p<0.05$

whereas our lupus nephritis model represents a chronic irreversible disease. In acute diseases, HMGB1 may act as an early alarmin that can initiate downstream proinflammatory signaling. In SLE, disease development occurs gradually and is multifactorial; hence, HMGB1 represents only one of the many proinflammatory factors that can activate the immune system and cause damage. This contention is supported by our finding that neutralization of HMGB1 does not halt or improve lupus nephritis development.

Inhibition of HMGB1 activity can be achieved in different ways. In our study, we used anti-HMGB1 antibodies to neutralize HMGB1. An alternative approach is to use the competitive receptor antagonist Box A to prevent binding of HMGB1 to its receptor receptor for advanced glycation end products (RAGE). In mouse models to contradict a recent study by Zhang et al. (36) showing that treatment with anti-HMGB1 antibodies reduced lupus-like disease, including proteinuria and glomerulonephritis, in lupus-prone BXSB mice (36). Lupus in BXSB mice is characterized by lymph node and spleen enlargement, hypergammaglobulinemia, anti-nuclear antibodies and immune complex glomerulonephritis, which usually becomes evident at around 5 months of age. A duplicated copy of the TLR7 gene is primarily responsible for the autoimmune phenotype. In MRL/lpr, we demonstrated that plasma HMGB1 levels are elevated upon disease development, but it is unclear whether the same holds true for the BXSB lupus mouse model. Moreover, the source of the anti-HMGB1 antibody used in the study 

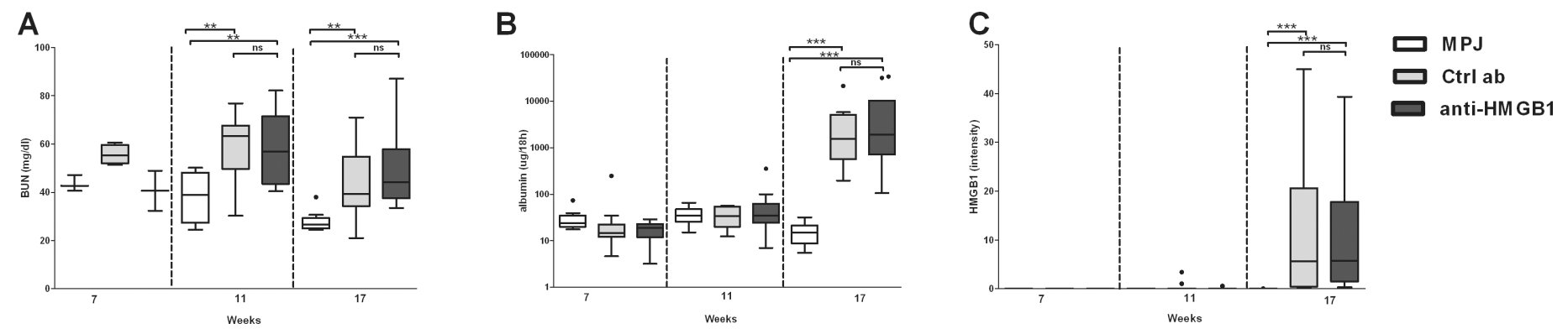

Figure 5. HMGB1 mAb treatment of MRL/Ipr mice does not affect albuminuria and HMGB1 urine levels. (A) Renal function was determined by measuring BUN levels at wks 7, 11 and 17. (B) Albumin was measured by ELISA in 18-h urine of MRL/MPJ $(n=10)$ and MRL/lpr $(n=10-12)$ mice at wks 7, 11 and 17. (C) HMGB1 was measured by Western blotting in 18-h urine of MRL/MPJ $(n=10)$ and MRL/lpr $(n=10-12)$ mice at wks 7, 11 and 17. HMGB1 levels are presented as values of fluorescence intensity and were normalized against a standard of Jurkat cell lysate. A Kruskal-Wallis test was used to test for overall differences between the three groups at each time point. To investigate which group was different, further testing was performed to compare groups separately using a Mann-Whitney $U$ test. Box and whiskers plot, median and interquartile range are shown for $n=8-12$ mice per group. Separate dots indicate outliers. ${ }^{* * *} p<0.0001$. ns, Nonsignificant.

A

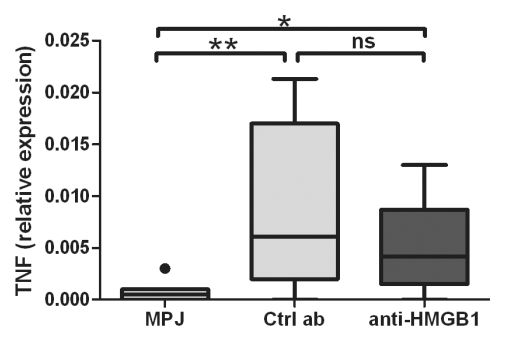

C

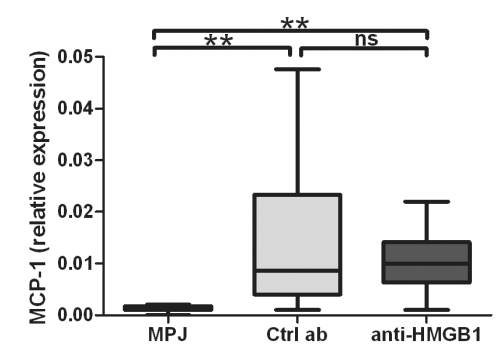

B

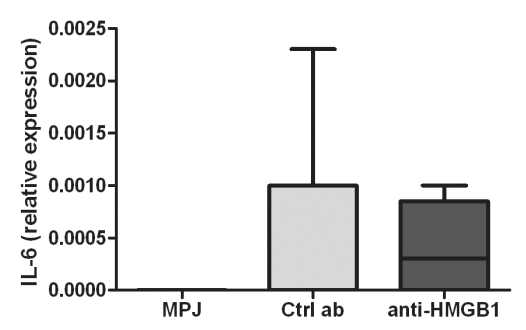

D

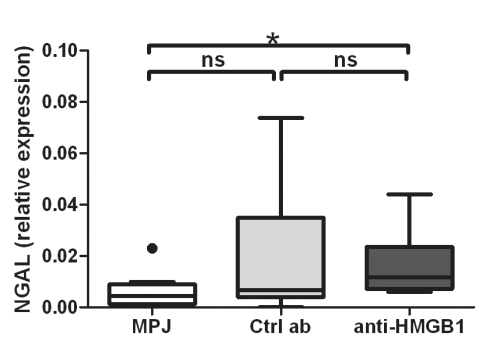

$\mathbf{E}$

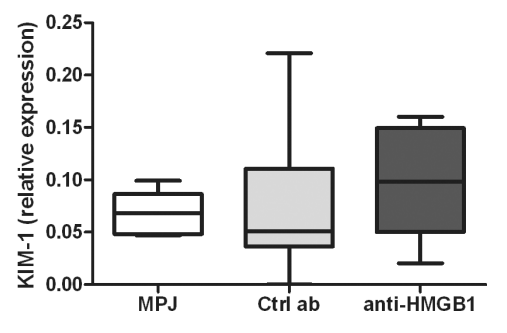

Figure 6. Anti-HMGBImAb treatment of MRL/Ipr mice does not affect renal mRNA levels of IL-6, TNF, MCP-1, NGAL or KIM-1. Expression levels of TNF- $\alpha$ (A), IL-6 (B), MCP-1 (C), NGAL (D) and KIM-1 (E) mRNA were analyzed in kidney tissues of MRL/MPJ mice ( $n=8)$ and MRL/lpr mice treated with control $(n=11)$ and mice treated with monoclonal anti-HMGBI $(n=9)$. Data are shown as relative expression compared with GAPDH. Box and whiskers plot, median and interquartile range are shown. Separate dots indicate outliers. A Kruskal-Wallis test was used to test for overall differences between the three groups. To investigate which group was different, further testing was performed to compare groups separately using a Mann-Whitney U test. ${ }^{*} p<0.05$; ${ }^{* *} p<0.01$. ns, Nonsignificant.

by Zhang et al. is different from ours, and a different treatment regimen was used that consisted of $10 \mu \mathrm{g} /$ mouse injected intraperitoneally three times a week from wk 16 until wk 26. Therefore, the differences between our study and the data from Zhang et al. might be explained by differences in mouse model, anti-HMGB1 antibody and treatment regimen. These inconclusive or contradictive results warrant further studies in different mouse models of SLE such as mice that lack MFGE8. 
Plasma HMGB1 levels, measured by Western blotting, were unaffected in MRL/lpr mice treated with neutralizing monoclonal anti-HMGB1 antibodies. We used Western blotting to detect HMGB1, since we and others have previously shown that serum proteins and antibodies interfere in the HMGB1 ELISA $(10,37)$. However, because of denaturation and high temperatures in Western blotting, immune complexes are dissociated, and the HMGB1 measured probably consists of free HMGB1 and previously complexed HMGB1. Our findings seem to imply that complexes of HMGB1-anti-HMGB1 that are formed during treatment are not cleared from the circulation, which can be explained by the fact that the antibody used is a neutralizing but not a depleting antibody.

MRL/lpr mice develop lymphadenopathy leading to the presence of autoreactive lymphocytes. Activated lymphocytes are a source of extracellular HMGB1 (38), which can contribute to high levels of HMGB1 in the spleen or lymph nodes. Therefore, because of high levels of HMGB1 locally, the dose of mAb $2 \mathrm{G} 7$ used here may have been insufficient to neutralize HMGB1 completely. Although we cannot exclude this possibility, previous studies in BXSB lupus mice and a sepsis model have clearly shown a positive effect of anti-HMGB1 antibody treatment, despite the development of splenomegaly $(21,36)$.

In untreated MRL/lpr mice, plasma levels of HMGB1 and anti-HMGB1 increase over time with disease progression, which is in line with data from SLE patients $(10,39)$. In the disease models described by Li et al. and Zhang et al., which do show a positive effect of blocking HMGB1, it is unclear whether anti-HMGB1 antibodies are also present $(33,36)$. At present, the role of endogenously formed anti-HMGB1 antibodies in disease pathogenesis is unclear and warrants further investigation. It is also possible that rheumatoid factors, which can be present in serum from MRL/lpr mice, can interfere with the activity of the anti-HMGB1 mAb. However, earlier studies using this antibody in animal models of rheumatoid arthritis did not observe a reduced effect of anti-HMGB1 $\mathrm{mAb}(7)$.

Because HMGB1 is known to induce proinflammatory cytokine release from immune cells, we also measured plasma proinflammatory cytokine levels. In vitro, HMGB1 has been shown to induce TNF- $\alpha$ release from mouse macrophages $(40,41)$ and IFN- $\alpha$ production from dendritic cells (42). In MRL/lpr mice, we observed increasing plasma levels of TNF- $\alpha$ and IFN- $\alpha$ over time. However, it is unclear whether these proinflammatory cytokines are induced solely by HMGB1 and/or are derived from autoreactive lymphocytes that are continuously present in MRL/lpr mice. Regardless of the source, we observed no effect of anti-HMGB1 antibody treatment on plasma proinflammatory cytokine levels.

Taken together, our data show that, although HMGB1 levels are increased in MRL/lpr mice, treatment with a monoclonal anti-HMGB1 antibody does not inhibit development or affect progression of lupus nephritis. Further studies are needed to validate these results in other mouse models of SLE.

\section{ACKNOWLEDGMENTS}

We thank Henk Moorlag, Berber Doornbos, Jun Yuan and Johan Bijzet for excellent technical support and Fiona Maas for help with the statistics. We thank Marius van den Heuvel for the evaluation and classification of the kidney slides. We also thank Kevin Tracey from the Feinstein Institute for Medical Research in New York for the generous gift of monoclonal anti-HMGB1 antibody (clone 2G7).

This work was supported by the Dutch Arthritis Foundation (grant number 11-1-401).

\section{DISCLOSURE}

The authors declare that they have no competing interests as defined by Molecular Medicine, or other interests that might be perceived to influence the results and discussion reported in this paper.

\section{REFERENCES}

1. Lisnevskaia L, Murphy G, Isenberg D. (2014) Systemic lupus erythematosus. Lancet. 384:1878-88.

2. Bianchi ME, Agresti A. (2005) HMG proteins: dynamic players in gene regulation and differentiation. Curr. Opin. Genet. Dev. 15:496-506.

3. Andersson U, Tracey KJ. (2011) HMGB1 is a therapeutic target for sterile inflammation and infection. Annu. Rev. Immunol. 29:139-62.

4. Taniguchi N, et al. (2003) High mobility group box chromosomal protein 1 plays a role in the pathogenesis of rheumatoid arthritis as a novel cytokine. Arthritis Rheum. 48:971-81.

5. Kokkola R, et al. (2002) High mobility group box chromosomal protein 1: a novel proinflammatory mediator in synovitis. Arthritis Rheum. 46:2598-603.

6. Wang H, et al. (1999) HMG-1 as a late mediator of endotoxin lethality in mice. Science. 285:248-51.

7. Schierbeck H, et al. (2011) Monoclonal anti-HMGB1 (high mobility group box chromosomal protein 1) antibody protection in two experimental arthritis models. Mol. Med. 17:1039-44.

8. Yang H, et al. (2004) Reversing established sepsis with antagonists of endogenous high-mobility group box 1. Proc. Natl. Acad. Sci. U. S. A. 101:296-301.

9. Shimazaki J, et al. (2012) Systemic involvement of high-mobility group box 1 protein and therapeutic effect of anti-high-mobility group box 1 protein antibody in a rat model of crush injury. Shock. 37:634-8.

10. Abdulahad DA, et al. (2011) High mobility group box 1 (HMGB1) and anti-HMGB1 antibodies and their relation to disease characteristics in systemic lupus erythematosus. Arthritis Res. Ther. 13:R71.

11. Abdulahad DA, et al. (2012) Urine levels of HMGB1 in systemic lupus erythematosus patients with and without renal manifestations. Arthritis Res. Ther. 14:R184.

12. Zickert A, et al. (2012) Renal expression and serum levels of high mobility group box 1 protein in lupus nephritis. Arthritis Res. Ther. 14:R36.

13. Li J, et al. (2010) Expression of high mobility group box chromosomal protein 1 and its modulating effects on downstream cytokines in systemic lupus erythematosus. J. Rheumatol. 37:766-75.

14. Ma C, et al. (2012) Elevated plasma level of HMGB1 is associated with disease activity and combined alterations with IFN-alpha and TNF-alpha in systemic lupus erythematosus. Rheumatol. Int. 32:395-402.

15. Kelley VE, Roths JB. (1985) Interaction of mutant lpr gene with background strain influences renal disease. Clin. Immunol. Immunopathol. 37:220-9.

16. Hicks J, Bullard DC. (2006) Review of autoimmune (lupus-like) glomerulonephritis in murine models. Ultrastruct. Pathol. 30:345-59. 
17. Theofilopoulos AN, Kono DH. (2001) Genetics of systemic autoimmunity and glomerulonephritis in mouse models of lupus. Nephrol. Dial. Transplant. 16 Suppl 6:65-7.

18. Li L, et al. (2012) Murine lupus strains differentially model unique facets of human lupus serology. Clin. Exp. Immunol. 168:178-85.

19. Yang H, et al. (2010) A critical cysteine is required for HMGB1 binding to toll-like receptor 4 and activation of macrophage cytokine release. Proc. Natl. Acad. Sci. U. S. A. 107:11942-7.

20. Ruan $X$, et al. (2015) Anti-HMGB1 monoclonal antibody ameliorates immunosuppression after peripheral tissue trauma: attenuated T-lymphocyte response and increased splenic CD11b (+) Gr-1 (+) myeloid-derived suppressor cells require HMGB1. Mediators Inflamm. 2015:458626.

21. Valdes-Ferrer SI, et al. (2013) HMGB1 mediates splenomegaly and expansion of splenic CD11b+ Ly-6C(high) inflammatory monocytes in murine sepsis survivors. J. Intern. Med. 274:381-90.

22. Wahamaa H, et al. (2007) HMGB1-secreting capacity of multiple cell lineages revealed by a novel HMGB1 ELISPOT assay. J. Leukoc. Biol. 81:129-36.

23. Yang H, et al. (2015) MD-2 is required for disulfide HMGB1-dependent TLR4 signaling. J. Exp. Med. 212:5-14.

24. Chavan SS, et al. (2012) HMGB1 mediates cognitive impairment in sepsis survivors. Mol. Med. 18:930-7.

25. Jager NA, et al. (2012) Targeted folate receptor beta fluorescence imaging as a measure of inflammation to estimate vulnerability within human atherosclerotic carotid plaque. J. Nucl. Med. 53:1222-9.

26. Weening JJ, et al. (2004) The classification of glomerulonephritis in systemic lupus erythematosus revisited. J. Am. Soc. Nephrol. 15:241-50.

27. Abdulahad DA, Westra J, Limburg PC, Kallenberg CGM, Bijl M. (2010) HMGB1 in systemic lupus erythematosus: its role in cutaneous lesions development. Autoimmun. Rev. 9:661-5.

28. Gao Q, et al. (2010) TLR4 mediates early graft failure after intraportal islet transplantation. Am. J. Transplant. 10:1588-96.

29. Qin S, et al. (2006) Role of HMGB1 in apoptosis-mediated sepsis lethality. J. Exp. Med. 203:1637-42.

30. Kojima M, et al. (2012) Role of high mobility group box chromosomal protein 1 in ischemia-reperfusion injury in the rat small intestine. J. Surg. Res. 178:466-71.

31. Kokkola R, et al. (2003) Successful treatment of collagen-induced arthritis in mice and rats by targeting extracellular high mobility group box chromosomal protein 1 activity. Arthritis Rheum. 48:2052-8.

32. Östberg T, et al. (2010) Protective targeting of high mobility group box chromosomal protein 1 in a spontaneous arthritis model. Arthritis Rheum. 62:2963-72.
33. Li X, Yue Y, Zhu Y, Xiong S. (2015) Extracellular, but not intracellular HMGB1, facilitates self-DNA induced macrophage activation via promoting DNA accumulation in endosomes and contributes to the pathogenesis of lupus nephritis. Mol. Immunol. 65:177-88.

34. Musumeci D, Roviello GN, Montesarchio D. (2014) An overview on HMGB1 inhibitors as potential therapeutic agents in HMGB1-related pathologies. Pharmacol. Ther. 141:347-57.

35. Mollica L, et al. (2007) Glycyrrhizin binds to high-mobility group box 1 protein and inhibits its cytokine activities. Chem. Biol. 14:431-41.

36. Zhang C, et al. (2014) High-mobility group box 1 inhibition alleviates lupus-like disease in BXSB mice. Scand. J. Immunol. 79:333-7.

37. Urbonaviciute $V$, et al. (2007) Factors masking HMGB1 in human serum and plasma. J. Leukoc. Biol. 81:67-74.

38. Li G, Liang X, Lotze MT. (2013) HMGB1: the central cytokine for all lymphoid cells. Front. Immunol. 4:68.

39. Hayashi A, et al. (2009) Lupus antibodies to the HMGB1 chromosomal protein: epitope mapping and association with disease activity. Mod. Rheumatol. 19:283-92.

40. Venereau E, et al. (2012) Mutually exclusive redox forms of HMGB1 promote cell recruitment or proinflammatory cytokine release. J. Exp. Med. 209:1519-28.

41. Yang H, et al. (2012) Redox modification of cysteine residues regulates the cytokine activity of high mobility group box-1 (HMGB1). Mol Med. 18:250-9.

42. Tian J, et al. (2007) Toll-like receptor 9-dependent activation by DNA-containing immune complexes is mediated by HMGB1 and RAGE. Nat. Immunol. 8:487-96.

Cite this article as: Schaper F, et al. (2016) Treatment with Anti-HMGB1 monoclonal antibody does not affect lupus nephritis in MRL/lpr mice. Mol. Med. 22:12-21. 